\title{
Interface tracking characteristics of color-gradient lattice Boltzmann model for immiscible fluids
}

\author{
A. Subhedar $\odot,{ }^{1, *}$ A. Reiter, ${ }^{1}$ M. Selzer, ${ }^{1,2}$ F. Varnik $\odot,{ }^{3}$ and B. Nestler ${ }^{1,2}$ \\ ${ }^{1}$ Institute for Digital Materials Science, Karlsruhe University of Applied Sciences, Moltkestraße 30, 76133 Karlsruhe, Germany \\ ${ }^{2}$ Institute of Applied Materials-Computational Materials Science, Karlsruhe Institute of Technology, \\ Straße am Forum 7, 76131 Karlsruhe, Germany \\ ${ }^{3}$ Interdisciplinary Centre for Advanced Materials Simulation, Ruhr-Universität Bochum, Universitätsstrasse 150, 44780 Bochum, Germany
}

(Received 5 August 2019; published 29 January 2020)

\begin{abstract}
We study the interface tracking characteristics of a color-gradient-based lattice Boltzmann model for immiscible flows. Investigation of the local density change in one of the fluid phases, via a Taylor series expansion of the recursive lattice Boltzmann equation, leads to the evolution equation of the order parameter that differentiates the fluids. It turns out that this interface evolution follows a conservative Allen-Cahn equation with a mobility which is independent of the fluid viscosities and surface tension. The mobility of the interface, which solely depends upon lattice speed of sound, can have a crucial effect on the physical dynamics of the interface. Further, we find that, when the equivalent lattice weights inside the segregation operator are modified, the resulting differential operators have a discretization error that is anisotropic to the leading order. As a consequence, the discretization errors in the segregation operator, which ensures a finite interface width, can act as a source of the spurious currents. These findings are supported with the help of numerical simulations.
\end{abstract}

DOI: 10.1103/PhysRevE.101.013313

\section{INTRODUCTION}

The lattice Boltzmann (LB) method has emerged as a powerful tool for the numerical study of multiphase fluid dynamical problems. A variety of multiphase LB models exist [1-4], each of them with strengths and weaknesses [5]. These models solve the discrete LB equation iteratively to ensure that the macroscopic bulk and boundary behavior of the fluid dynamical quantities, such as density, velocity, and stress tensor, is recovered. The interface between fluids is typically represented through a diffuse interface directly or indirectly for numerical convenience. Such a diffuse representation simplifies evaluation of the curvature and facilitates tracking of the interface.

Among these models, the color gradient (CG) or the chromo-dynamic method [2] is an attractive choice as it allows one to tune the physical properties of fluids like density, viscosity, and surface tension independently of each other. Various improvements have been made in the original CG model [2], which include reduction of the lattice pinning effects [6], generalization of the surface tension term for rectangular lattices [7], momentum correction term for unequal density case [8-10], extension to multiple-relaxationtime (MRT) method [9], and fluid-solid wetting boundary conditions [11]. The CG model typically uses the colored fluid convention to enumerate and an order parameter in the form of a phase field variable to differentiate between the fluid states. The microscopic fluid populations are redistributed in a direction normal to the interface between fluids to ensure that they do not mix together (known as the recoloring step). The information of the local interface normal is then used to add a

*amol.subhedar@hs-karlsruhe.de term amounting to surface tension, known as the perturbation step.

Different CG models have been applied successfully in the numerical study of unsteady and steady fluid problems [12-15], and some efforts have been made in order to understand the nature of the interface tracking involved in these models. Although introduced for numerical convenience, the auxiliary diffuse interface width adds a new length scale and therefore a new timescale to the system. It is important to know the nature of these scales and to make sure that they comply with the macroscopic mass and momentum balance boundary conditions at the interface between fluids. Kehrwald was one of first to identify [16] that the CG interface tracking scheme is closely related to the conservative volume tracking method. This study used a suboptimal numerical segregation scheme where fluid densities are redistributed to minimize the diffusion of color across the interface [17]. Such an approach, however, is susceptible to the lattice pinning artifact [6]. The equilibrium phase field profile, and consequently the width of the diffuse interface, was obtained by Latva-Kokko and Rothman for a case of planar interface at the steady state [6]. Hollis et al. showed [18] that the interface dynamics follows an advection type of equation with a source term that depends upon the external forces, density gradients, and fluid velocity. To identify the length scale of the interface, however, they resorted to consideration of a steady-state case. This steady-state analysis was later generalized to include curved interfaces [19]. Recently, Burgin et al. [20] derived evolution equations for the individual fluid density fields, where a density contrast between the two fluids is accounted for.

In the present work, we shed more light on this issue. More specifically, we show that a unified characteristic equation of the phase field dynamics, which correctly identifies the 
relevant length scale of the interface, can be obtained within a single framework. This phase field dynamics follows a conservative Allen-Cahn type of equation where the phase field mobility $M$, which controls the relaxation rate, is determined solely by the lattice speed of sound. A similar type of equation is used to track the interface in other multiphase fluid numerical methods $[21,22]$. To this end, we analyze the density change of one of the fluids in the interfacial area using the segregation operator proposed by Latva-Kokko and Rothman [6] (operator I) and a slightly modified version of it by Halliday et al. [23] (operator II). We use a recursive representation of the $\mathrm{LB}$ equation [24] to obtain information on the nonequilibrium part of the fluid populations. Individual terms that appear in the density exchange of either of the fluids are then Taylor expanded up to second order in spatial discretization. Although the terms in the third and higher orders of spatial derivatives are neglected, we make sure that these error terms are spatially isotropic to the leading order. For the present analysis, it is assumed that the reference densities and viscosities of the two fluids are the same.

Further, we find that the segregation operator I leads to discretization errors that are anisotropic in the third order of spatial derivatives. The modified version of it, operator II, which was proposed with the intention of making the Chapman-Enskog analysis more amenable [23], is free of such discretization errors. These discretization errors are one of the sources of the spurious currents. Thus these currents are weaker for the operator II than the operator I as we show in the following. An additional advantage of operator II is that the interfacial width remains the same for all the rectangular lattices in two and three dimensions. The downside is that the interface cannot be made as sharp as the one obtained using operator I without inducing unphysical fluid densities [23].

The findings of the present analysis are verified against numerical simulations. To highlight the Allen-Cahn type nature of the CG phase field equation, Zalesak's test [25] is performed where external velocity drives the fluid flow. The phase field mobility $M$, the coefficient of the Laplacian term in the Allen-Cahn equation, has an important influence on the interface shape in this case. We compare the present $\mathrm{CG}$ model and direct numerical solution of the corresponding Allen-Cahn equation for a range of viscosities and discuss its effect upon the phase field mobility. Finally, in a view of discretization errors induced, we discuss the reduction in the spurious currents when segregation operator II is used instead of I.

\section{THE TWO-PHASE CG MODEL}

The evolution equations for the two-phase color gradient method are based upon fluid distributions $f_{k, i}$, where $i$ and $k$ denote lattice direction and phase index $(k$ is either 1 or 2). The number of lattice directions depends upon the spatial dimensions and choice of the lattice. For the sake of definiteness, we choose the D2Q9 lattice [26]. The total fluid populations, $N_{i}=f_{1, i}+f_{2, i}$, undergo a local collision step that includes the surface tension effect in the following way:

$$
N_{i}^{*}=\left(1-\frac{\delta t}{\tau}\right) N_{i}+\frac{\delta t}{\tau} N_{i}^{\mathrm{eq}}+F_{i},
$$

where $N_{i}^{*}$ are total postcollision populations, the time step $\delta t=1$, and $\tau$ is the relaxation time that depends upon effective kinematic viscosity $v$ as $\tau=\frac{\delta t}{2}+\frac{v}{c_{\mathrm{s}}^{2}}$. For rectangular lattices like D2Q9 and D3Q19, the lattice speed of sound $c_{\mathrm{s}}$ turns out to be $\frac{1}{\sqrt{3}}$ in lattice units (lu) [27]. The term $F_{i}$ on the right-hand side of Eq. (1) accounts for the macroscopic external forces $\mathbf{F}$ in the fluid. We have used the BhatnagarGross-Krook (BGK) approximation [28] in Eq. (1) to keep the present analysis tractable. The equilibrium distribution function $N_{i}^{\mathrm{eq}}$ in Eq. (1) is given by

$$
N_{i}^{\mathrm{eq}}=\rho w_{i}\left[1+\frac{\mathbf{c}_{i} \cdot \mathbf{u}}{c_{\mathrm{s}}^{2}}+\frac{\left(\mathbf{c}_{i} \cdot \mathbf{u}\right)^{2}}{2 c_{\mathrm{s}}^{4}}-\frac{\mathbf{u}^{2}}{2 c_{\mathrm{s}}^{2}}\right],
$$

where $w_{i}$ is weight in the lattice direction $i$ with $w_{0}=\frac{4}{9}$, $w_{1-4}=\frac{1}{9}$, and $w_{5-9}=\frac{1}{36}$. The lattice velocities $\mathbf{c}_{i}$ are defined as $\mathbf{c}_{0}=(0,0), \mathbf{c}_{1,3}=( \pm 1,0), \mathbf{c}_{2,4}=(0, \pm 1), \mathbf{c}_{5,6}=( \pm 1,1)$, and $\mathbf{c}_{7,8}=( \pm 1,-1)$. The densities of the fluids are denoted by $\rho_{1}$ and $\rho_{2}$, respectively, while the total density is $\rho=$ $\rho_{1}+\rho_{2}$. The two phases present are differentiated by the phase field variable $\varphi$ using difference of the densities as

$$
\varphi=\frac{\rho_{1}-\rho_{2}}{\rho_{1}+\rho_{2}} .
$$

A unit vector in the direction of the phase field gradient $\hat{\mathbf{n}}=\frac{\nabla \varphi}{|\nabla \varphi|}$ defines the unit normal to the interface. The total fluid density and velocity are evaluated by taking the zeroth and first moment of the fluid populations with respect to lattice velocity $\mathbf{c}_{i}$ as

$$
\rho=\sum_{i}\left(f_{1, i}+f_{2, i}\right)
$$

and

$$
\rho \mathbf{u}=\sum_{i}\left(f_{1, i}+f_{2, i}\right) \mathbf{c}_{i} .
$$

The form of $F_{i}$ within the exact difference scheme [29] is given as

$$
\begin{aligned}
F_{i} & =N_{i}^{\mathrm{eq}}\left(\rho, \mathbf{u}+\frac{\mathbf{F} \delta t}{\rho}\right)-N_{i}^{\mathrm{eq}}(\rho, \mathbf{u}) \\
& =w_{i} \delta t\left[\frac{\mathbf{F} \cdot \mathbf{c}_{i}}{c_{\mathrm{s}}^{2}}+\frac{(\mathbf{v} \mathbf{F}+\mathbf{F v}): \mathbf{Q}_{i}}{2 c_{\mathrm{s}}^{4}}\right],
\end{aligned}
$$

where the tensor $\mathbf{Q}_{i}$ stands for $\mathbf{c}_{i} \mathbf{c}_{i}-c_{\mathrm{s}}^{2} \mathbf{I}, \mathbf{I}$ is a secondorder identity tensor, the notation ":" stands for the tensor contraction, and $\mathbf{v}$ is the actual macroscopic fluid velocity. In the presence of forces it is given as

$$
\mathbf{v}=\mathbf{u}+\frac{\mathbf{F} \delta t}{2 \rho},
$$

where the second term on the right-hand side of Eq. (7) is a correction, necessary in order to accurately impose spatially varying external forces. The surface tension term $\mathbf{F}$, in the form of external force, is defined as

$$
\mathbf{F}=\frac{1}{2} \sigma \kappa \nabla \varphi,
$$

where $\sigma$ is the surface tension, $\kappa$ is the interface curvature, and the factor $\frac{1}{2}$ accounts for the variation of $\varphi$ in the range $(-1,1)$. Addition of such a term is not sufficient to maintain 
a stable interface between the fluids. For this reason, an additional step, called the recoloring or segregation step, is necessary to ensure a finite interface thickness. In the original work of D'Ortona et al. [30] and later adopted by LatvaKokko and Rothman [6], the scheme for fluid segregation (operator I) is defined as

$$
\begin{aligned}
& f_{1, i}^{*}(\mathbf{x}, t)=\frac{\rho_{1}}{\rho} N_{i}^{*}+\delta t \beta \frac{\rho_{1} \rho_{2}}{\rho^{2}} \frac{\nabla \varphi \cdot \mathbf{c}_{i}}{\left|\nabla \varphi \| \mathbf{c}_{i}\right|} N_{i}^{\mathrm{eq}}{ }^{\mathrm{q}=0}, \\
& f_{2, i}^{*}(\mathbf{x}, t)=\frac{\rho_{2}}{\rho} N_{i}^{*}-\delta t \beta \frac{\rho_{1} \rho_{2}}{\rho^{2}} \frac{\nabla \varphi \cdot \mathbf{c}_{i}}{\left|\nabla \varphi \| \mathbf{c}_{i}\right|} N_{i}^{\mathrm{eq}}{ }^{\mathrm{q}=0},
\end{aligned}
$$

where $f_{1, i}^{*}, f_{2, i}^{*}$ are individual postcollision fluid populations and $N_{i}^{\mathrm{eq} \mathrm{u}=0}$ is the total equilibrium populations evaluated for zero fluid velocity. Equation (9) is defined for all the lattice velocities, except for the rest populations with $\mathbf{c}_{i}=0$. In this case, the second terms on the right-hand side of Eq. (9) are defined to be zero. Latva-Kokko and Rothman have shown that for a one-dimensional case the parameter $\beta$ is inversely proportional to the interface width [6]. The segregation scheme (operator II) proposed by Halliday et al. [23] is defined as follows:

$$
\begin{aligned}
& f_{1, i}^{*}(\mathbf{x}, t)=\frac{\rho_{1}}{\rho} N_{i}^{*}+\delta t \beta \frac{\rho_{1} \rho_{2}}{\rho^{2}} \frac{\nabla \varphi \cdot \mathbf{c}_{i}}{|\nabla \varphi|} N_{i}^{\mathrm{eq}}{ }^{\mathrm{u}=0}, \\
& f_{2, i}^{*}(\mathbf{x}, t)=\frac{\rho_{2}}{\rho} N_{i}^{*}-\delta t \beta \frac{\rho_{1} \rho_{2}}{\rho^{2}} \frac{\nabla \varphi \cdot \mathbf{c}_{i}}{|\nabla \varphi|} N_{i}^{\mathrm{eq}},
\end{aligned}
$$

where in comparison with Eq. (9) a factor $\left|\mathbf{c}_{i}\right|$ is missing in the denominator of second term on the right-hand side. From the point of view of dimensional consistency, $\left|\mathbf{c}_{i}\right|$ should be treated as a numerical factor instead of a term with dimensions of speed. This modification changes the effective lattice weights for lattice directions where $\left|\mathbf{c}_{i}\right| \neq 1$. Given that lattice weights $w_{i}$ preserve isotropy of discrete space up to fourth order in the lattice tensors [31], the resulting differential operators with the modified lattice weights suffer from discretization errors. It is to be noted that we have included a factor of $\delta t$ in the second term on the right-hand sides of Eq. (9) and Eq. (10). As we show below, this construction makes it easier to identify $\beta$ as the inverse of length scale that characterizes the interface.

The general CG scheme is completed with the propagation step as follows:

$$
f_{k, i}\left(\mathbf{x}+\mathbf{c}_{i} \delta t, t+\delta t\right)=f_{k, i}^{*}(\mathbf{x}, t) .
$$

$N_{i}^{\mathrm{eq}}$ and $F_{i}$ in Eq. (9) can be expressed in terms of the local fluid dynamical quantities and their spatial derivatives. Our aim here is to obtain an expression for $N_{i}$ in terms of $N_{i}^{\mathrm{eq}}$ and the surface tension term $F_{i}$. We note that as long as the interface is tracked appropriately, it is sufficient to solve LB equation for total fluid populations $N_{i}$ alone. Given that the segregation step [Eq. (9) or Eq. (10)] takes care of the interface tracking, we rewrite Eq. (1) as

$$
\begin{aligned}
N_{i}(\mathbf{x}, t)= & \left(1-\frac{\delta t}{\tau}\right) N_{i}\left(\mathbf{x}-\mathbf{c}_{i} \delta t, t-\delta t\right) \\
& +\frac{\delta t}{\tau} N_{i}^{\mathrm{eq}}\left(\mathbf{x}-\mathbf{c}_{i} \delta t, t-\delta t\right)+F_{i}\left(\mathbf{x}-\mathbf{c}_{i} \delta t, t-\delta t\right) .
\end{aligned}
$$

To express $N_{i}$ in terms of the total equilibrium populations and surface tension terms, we follow the procedure outlined by Holdych et al. [24,32]. We write Eq. (12) as

$$
\begin{aligned}
& N_{i}\left(\mathbf{x}-n \mathbf{c}_{i} \delta t, t-n \delta t\right) \\
& =\left(1-\frac{\delta t}{\tau}\right) N_{i}\left[\mathbf{x}-(n+1) \mathbf{c}_{i} \delta t, t-(n+1) \delta t\right] \\
& \quad+\frac{\delta t}{\tau} N_{i}^{\mathrm{eq}}\left[\mathbf{x}-(n+1) \mathbf{c}_{i} \delta t, t-(n+1) \delta t\right] \\
& \quad+F_{i}\left[\mathbf{x}-(n+1) \mathbf{c}_{i} \delta t, t-(n+1) \delta t\right],
\end{aligned}
$$

where $n$ is a non-negative integer. Multiplying Eq. (13) with $\left(1-\frac{\delta t}{\tau}\right)^{n}$ for increasing values of $n$ and adding them to Eq. (12), we obtain

$$
\begin{aligned}
N_{i}(\mathbf{x}, t)= & \frac{\delta t}{\tau} \sum_{n=1}^{\infty}\left(1-\frac{\delta t}{\tau}\right)^{n-1} N_{i}^{\mathrm{eq}}\left(\mathbf{x}-n \mathbf{c}_{i} \delta t, t-n \delta t\right) \\
& +\sum_{n=1}^{\infty}\left(1-\frac{\delta t}{\tau}\right)^{n-1} F_{i}\left(\mathbf{x}-n \mathbf{c}_{i} \delta t, t-n \delta t\right),
\end{aligned}
$$

where we implicitly assumed that $\lim _{n \rightarrow \infty}\left(1-\frac{\delta t}{\tau}\right)^{n} \rightarrow 0$, or $\tau>\frac{\delta t}{2}$. Now, a first-order Taylor expansion of $N_{i}^{\text {eq }}$ and $F_{i}$ in Eq. (14) around position and time $(\mathbf{x}, \mathrm{t})$ gives

$$
\begin{aligned}
N_{i}(\mathbf{x}, t)= & N_{i}^{\mathrm{eq}}(\mathbf{x}, t)-\tau\left(\frac{\partial}{\partial t}+\mathbf{c}_{i} \cdot \nabla\right) N_{i}^{\mathrm{eq}}(\mathbf{x}, t) \\
& +\frac{\tau}{\delta t} F_{i}(\mathbf{x}, t)-\frac{\tau^{2}}{\delta t}\left(\frac{\partial}{\partial t}+\mathbf{c}_{i} \cdot \nabla\right) F_{i}(\mathbf{x}, t) .
\end{aligned}
$$

The deviation of $N_{i}$ from $N_{i}^{\mathrm{eq}}, N_{i}^{\mathrm{neq}}=N_{i}-N_{i}^{\mathrm{eq}}$, is then written as

$$
\begin{aligned}
N_{i}^{\mathrm{neq}}(\mathbf{x}, t)= & -\tau\left(\frac{\partial}{\partial t}+\mathbf{c}_{i} \cdot \nabla\right) N_{i}^{\mathrm{eq}}(\mathbf{x}, t) \\
& +\frac{\tau}{\delta t} F_{i}(\mathbf{x}, t)-\frac{\tau^{2}}{\delta t}\left(\frac{\partial}{\partial t}+\mathbf{c}_{i} \cdot \nabla\right) F_{i}(\mathbf{x}, t) .
\end{aligned}
$$

Equation (16) will be used for analyzing the local density exchange of the first fluid in the following. We emphasis that the Taylor expansion utilized in Eq. (15) is based upon the assumption that the spatial and temporal derivatives are small or alternatively $\delta t$ is sufficiently small. For the sake of convenience, we have set the time step $\delta t$ to unity. Thus, we treat derivatives as an effective smallness parameter [33] in the present work. Using Eq. (16), the postcollision fluid populations for the first fluid can be written as

$$
\begin{aligned}
f_{1, i}^{*} & =\frac{\rho_{1}}{\rho}\left[\left(1-\frac{\delta t}{\tau}\right) N_{i}+\frac{\delta t}{\tau} N_{i}^{\mathrm{eq}}\right]+\beta \delta t \frac{\rho_{1} \rho_{2}}{\rho^{2}} \frac{\mathbf{c}_{i} \cdot \hat{\mathbf{n}}}{\left|\mathbf{c}_{i}\right|} N_{i}^{\mathrm{eq}}{ }^{\mathrm{u}=0} \\
& =f_{1, i}^{\mathrm{eq}}+\frac{\rho_{1}}{\rho}\left(1-\frac{\delta t}{\tau}\right) N_{i}^{\mathrm{neq}}+\beta \delta t \frac{\rho_{1} \rho_{2}}{\rho^{2}} \frac{\mathbf{c}_{i} \cdot \hat{\mathbf{n}}}{\left|\mathbf{c}_{i}\right|} N_{i}^{\mathrm{eq}} \cdot(1)=0
\end{aligned}
$$


(a)

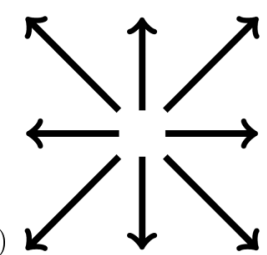

(b)

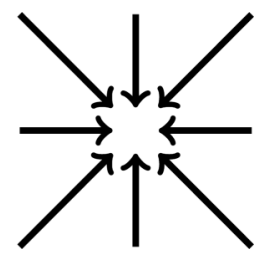

FIG. 1. Postcollision populations of the first fluid $f_{1, i}^{*}$ (a) leaving and (b) arriving at any node $\mathbf{x}$ for a D2Q9 lattice during the propagation step. The net difference of these populations gives rise to a change in the density of the first fluid.

We regroup the postcollision populations of the first fluid on the right-hand side of Eq. (17) as

$$
\begin{gathered}
f_{1, i}^{*, \mathrm{eq}}=\rho_{1} w_{i}\left(1+\frac{\mathbf{c}_{i} \cdot \mathbf{u}}{c_{\mathrm{s}}^{2}}+\frac{\mathbf{u u}: \mathbf{Q}_{i}}{2 c_{\mathrm{s}}^{4}}\right) \\
f_{1, i}^{*,(1)}=-\frac{\rho_{1}}{\rho}(\tau-\delta t) D_{i} \rho w_{i}\left(1+\frac{\mathbf{c}_{i} \cdot \mathbf{u}}{c_{\mathrm{s}}^{2}}+\frac{\mathbf{u u}: \mathbf{Q}_{i}}{2 c_{\mathrm{s}}^{2}}\right) \\
+\frac{\rho_{1}}{\rho} \frac{\tau-\delta t}{\delta t} F_{i}-\frac{\rho_{1}}{\rho} \frac{\tau-\delta t}{\delta t} \tau D_{i} F_{i} \\
f_{1, i}^{*, \mathrm{seg}}=\beta \delta t \frac{\rho_{1} \rho_{2}}{\rho^{2}} \frac{\mathbf{c}_{i} \cdot \hat{\mathbf{n}}}{\left|\mathbf{c}_{i}\right|} N_{i}^{\mathrm{eq}}{ }^{\mathrm{u}=0}
\end{gathered}
$$

where $D_{i}=\frac{\partial}{\partial t}+\mathbf{c}_{i} \cdot \nabla$ is the total lattice derivative and we have substituted Eq. (16) in Eq. (17).

\section{TEMPORAL EVOLUTION OF THE INTERFACE}

For the sake of clarity, we analyze the change in density of the first fluid due to the propagation step. Equation (17) gives the expression for the collided populations of the first fluid at any lattice node. The density change of the first fluid in a unit time step $\delta t$ then can be written as (see Fig. 1)

$$
\delta \rho_{1}(\mathbf{x}, t)=-\sum_{i} f_{1, i}^{*}(\mathbf{x}, t)+\sum_{i} f_{1, i}^{*}\left(\mathbf{x}-\mathbf{c}_{i} \delta t, t\right) .
$$

The order of differential terms now denotes the order of smallness in $\delta t$ and neglecting the third and higher order in spatial derivatives is equivalent to neglecting the terms in $O\left(\delta t^{3}\right)$.

To derive the equation for the phase field variable given by Eq. (3), we analyze the exchange of the postcollision fluid populations $f_{1, i}^{*}$ by substituting Eq. (18), Eq. (19), and Eq. (20) in Eq. (21). The necessary expressions of density exchange, obtained via the Taylor expansion, are given in the Appendix.

\section{A. Contribution of equilibrium populations}

The first term in Eq. (18) is of the form $w_{i} \rho_{1}$. After the propagation step, the change in the density of first fluid due to this term can be found by substituting this term in Eq. (21) and using Eq. (A1) to yield $\frac{c_{\mathrm{s}}^{2} \delta t^{2}}{2} \nabla^{2} \rho_{1}$. The second term on the right-hand side of Eq. (18) can be identified as $w_{i} \frac{\rho_{1} \mathbf{u} \cdot \mathbf{c}_{i}}{c_{\mathrm{S}}^{2}}$. From Eq. (A2) the change in density due to this term can be written as $-\delta t \nabla \cdot\left(\rho_{1} \mathbf{u}\right)$. The third and fourth terms can be identified as $w_{i} \frac{\left(\mathbf{c}_{i} \cdot \rho_{1} \mathbf{u}\right)\left(\mathbf{c}_{i} \cdot \mathbf{u}\right)}{2 c_{\mathrm{S}}^{4}}-w_{i} \frac{\rho_{1} \mathbf{u} \cdot \mathbf{u}}{2 c_{\mathrm{S}}^{2}}$. From Eq. (A4) and Eq. (A1), the net effect of this term is $\frac{\delta t^{2}}{2} \nabla \cdot \nabla \cdot\left(\rho_{1} \mathbf{u u}\right)$. Thus, the total

change in the density of the first fluid due to the propagation of the equilibrium fluid populations, in a unit time step $\delta t$, is

$$
\delta \rho_{1}^{\mathrm{eq}}=\frac{c_{\mathrm{s}}^{2} \delta t^{2}}{2} \nabla^{2} \rho_{1}-\delta t \nabla \cdot \rho_{1} \mathbf{u}+\frac{\delta t^{2}}{2} \nabla \cdot\left[\nabla \cdot\left(\rho_{1} \mathbf{u u}\right)\right] .
$$

Division of both sides of Eq. (22) by $\delta t(=1)$ and assuming that all the relevant physical timescales are much larger than $\delta t$, we replace $\frac{\delta \rho_{1}^{\text {eq }}}{\delta t}$ by $\frac{\partial \rho_{1}^{\text {eq }}}{\partial t}$. Thus, Eq. (22) now reads

$$
\frac{\partial \rho_{1}^{\mathrm{eq}}}{\partial t}=\frac{c_{\mathrm{s}}^{2} \delta t}{2} \nabla^{2} \rho_{1}-\nabla \cdot \rho_{1} \mathbf{u}+\frac{\delta t}{2} \nabla \cdot\left[\nabla \cdot\left(\rho_{1} \mathbf{u u}\right)\right] .
$$

For the other contributions to the density change, a similar procedure is followed.

\section{B. Contribution of nonequilibrium populations}

From the case of single-phase fluid, we know that the nonequilibrium fluid populations do not contribute to the density of the fluid. These nonequilibrium populations generally contain information on the shear stress tensor [27,34]. For the case at hand of two-phase fluids, we show that the nonequilibrium fluid populations also do not have an effect upon the continuity equation of either fluid phase, up to the terms that are in the second order of spatial derivatives.

The nonequilibrium term containing a time derivative of $N_{i}^{\mathrm{eq}}$ in Eq. (19) is $-\frac{\rho_{1}}{\rho}(\tau-\delta t) \frac{\partial}{\partial t} N_{i}^{\mathrm{eq}}$. The net contribution of this term can be found using Eq. (A1), Eq. (A2), and Eq. (A4) to yield

$$
\begin{aligned}
\frac{1}{\tau-\delta t} \frac{\partial}{\partial t} \rho_{1}^{\text {neq, } \partial_{t}}= & -\frac{c_{\mathrm{s}}^{2} \delta t}{2} \nabla^{2}\left[\frac{\rho_{1}}{\rho} \frac{\partial \rho}{\partial t}\right]+\nabla \cdot\left(\frac{\rho_{1}}{\rho} \frac{\partial}{\partial t} \rho \mathbf{u}\right) \\
& +\frac{\delta t}{2} \nabla \cdot\left(\nabla \cdot \frac{\rho_{1}}{\rho} \frac{\partial}{\partial t} \rho \mathbf{u u}\right) \\
= & \nabla \cdot\left(\frac{\rho_{1}}{\rho} \frac{\partial}{\partial t} \rho \mathbf{u}\right)
\end{aligned}
$$

where third- and higher-order derivatives are not taken into account.

The nonequilibrium term containing spatial derivatives of $N_{i}^{\mathrm{eq}}$ in Eq. (19) is $-\frac{\rho_{1}}{\rho}(\tau-\delta t) \mathbf{c}_{i} \cdot \nabla N_{i}^{\mathrm{eq}}$. From Eq. (A5), Eq. (A6), and Eq. (A7) one can write

$$
\frac{\partial}{\partial t} \rho_{1}^{\text {neq, } \mathbf{c}_{i} \cdot \nabla}=(\tau-\delta t) \nabla \cdot\left[\frac{\rho_{1}}{\rho}\left(\nabla \rho c_{\mathrm{s}}^{2}+\nabla \cdot \rho \mathbf{u u}\right)\right],
$$

where, again, the third-order derivatives are neglected.

The surface tension term in the form of external force is given as $\frac{\tau-\delta t}{\delta t} \frac{\rho_{1}}{\rho} F_{i}$ in Eq. (19). The form of $F_{i}$ in the present work is given by Eq. (6). Utilizing Eq. (A2) and Eq. (A7) together with Eq. (6) yields

$$
\frac{\partial}{\partial t} \rho_{1}^{\text {neq, } \sigma}=(\tau-\delta t) \nabla \cdot\left[-\frac{\rho_{1}}{\rho} \mathbf{F}+\delta t \frac{\rho_{1}}{\rho} \nabla \cdot \boldsymbol{\Psi}\right],
$$

where we have used $\boldsymbol{\Psi}=\frac{\mathbf{F v}+\mathbf{v F}}{2}$ for brevity. The interfacial surface tension force term, $\mathbf{F}$, contains second-order spatial derivatives of the phase field variable. In view of this fact, we do not consider additional surface tension term involving a total lattice derivative in Eq. (19). 
The net contribution of the nonequilibrium terms thus can be added by Eq. (24), Eq. (25), and Eq. (26) as

$$
\begin{aligned}
\frac{\partial \rho_{1}^{\text {neq }}}{\partial t}= & \frac{\partial}{\partial t} \rho_{1}^{\text {neq, } \partial_{t}}+\frac{\partial}{\partial t} \rho_{1}^{\text {neq, } \mathbf{c}_{i} \cdot \nabla}+\frac{\partial}{\partial t} \rho_{1}^{\text {neq, } \sigma} \\
= & (\tau-\delta t) \nabla \cdot\left[\frac{\rho_{1}}{\rho}\left[\nabla \rho c_{\mathrm{s}}^{2}-\mathbf{F}+\nabla \cdot \rho \mathbf{u u}+\partial_{t} \rho \mathbf{u}\right]\right] \\
& +(\tau-\delta t) \delta t \boldsymbol{\nabla} \cdot\left[\frac{\rho_{1}}{\rho} \nabla \cdot \boldsymbol{\Psi}\right]
\end{aligned}
$$

where $\partial_{t}$ is the partial derivative with respect to time.

\section{Contribution of segregation populations}

Now, we look into the segregation operator contribution that is responsible for maintaining a finite width of the interface. On the right-hand side of Eq. (20), $N_{i}^{\mathrm{eq}}$ when evaluated for identically zero fluid velocity is simply equal to $w_{i} \rho$ [see Eq. (2)]. Given that in Eq. (20) the unit interface normal has a dot product with a unit lattice velocity $\frac{\mathbf{c}_{i}}{\left|\mathbf{c}_{i}\right|}\left(\left|\mathbf{c}_{i}\right| \neq 0\right)$, the corresponding lattice weights $w_{i}^{\text {aniso }}$ change accordingly. For the D2Q9 lattice, only the lattice directions such that $\left|\mathbf{c}_{i}\right|=\sqrt{2}$ are affected by this construction. Specifically, these weights are $w_{0}^{\text {aniso }}=\frac{4}{9}$ and $w_{1-4}^{\text {aniso }}=\frac{1}{9}$ and $w_{5-8}^{\text {aniso }}=\frac{1}{36 \sqrt{2}}$. Now we identify the right-hand side of Eq. (20) as $w_{i}^{\text {aniso } \frac{\psi}{\psi} \cdot \mathbf{c}_{i}} c_{\mathrm{s}}^{2}$ with $\boldsymbol{\psi}=\delta t \beta c_{\mathrm{s}}^{2} \rho \frac{\rho_{1} \rho_{2}}{\rho^{2}} \hat{\mathbf{n}}$. From Eq. (A3) it follows that the net change in density of the first fluid can be written as

$$
\begin{aligned}
\frac{\partial}{\partial t} \rho_{1}^{\mathrm{seg}}= & -\frac{4+\sqrt{2}}{6} \nabla \cdot\left(\beta c_{\mathrm{s}}^{2} \rho \frac{\rho_{1} \rho_{2}}{\rho^{2}} \hat{\mathbf{n}}\right) \delta t \\
& -\frac{4+\sqrt{2}}{36}\left(\frac{\partial^{3} \psi_{x}}{\partial x^{3}}+\frac{\partial^{3} \psi_{y}}{\partial y^{3}}\right) \delta t^{3} \\
& -\frac{3 \sqrt{2}}{36}\left(\frac{\partial}{\partial x} \frac{\partial^{2} \psi_{x}}{\partial y^{2}}+\frac{\partial}{\partial y} \frac{\partial^{2} \psi_{y}}{\partial x^{2}}\right) \delta t^{3},
\end{aligned}
$$

where $\psi_{x}$ and $\psi_{y}$ denote Cartesian components of the vector $\boldsymbol{\psi}$. Although we include only the terms up to the second order in spatial derivatives, as far as possible, it is desirable to have the leading-order error term to be spatially isotropic. Equation (28) shows that the leading-order error term is, in fact, anisotropic. A simple remedy for this problem is to use the segregation operator II, Eq. (10), where $\left|\mathbf{c}_{i}\right|$ is removed from the denominator. The use of this segregation operator along with Eq. (A2) now results in

$$
\frac{\partial}{\partial t} \rho_{1}^{\mathrm{seg}}=-\nabla \cdot\left[1+\frac{\delta t^{2} c_{\mathrm{s}}^{2}}{2} \nabla^{2}\right]\left(\beta c_{\mathrm{s}}^{2} \rho \frac{\rho_{1} \rho_{2}}{\rho^{2}} \hat{\mathbf{n}}\right) \delta t,
$$

where the isotropy of the differential operators is now apparent. We proceed with neglecting the third-order terms and write

$$
\frac{\partial}{\partial t} \rho_{1}^{\mathrm{seg}}=-\nabla \cdot\left(\beta c_{\mathrm{s}}^{2} \rho \frac{\rho_{1} \rho_{2}}{\rho^{2}} \hat{\mathbf{n}}\right) \delta t .
$$

Now that all the different contributions to the density change of the first fluid are determined, the total change in the density of the first fluid can be written from Eq. (23), Eq. (27), and Eq. (30) as

$$
\begin{aligned}
\frac{\partial \rho_{1}}{\partial t}+\nabla \cdot \rho_{1} \mathbf{u} \\
=\frac{c_{\mathrm{s}}^{2} \delta t}{2} \nabla^{2} \rho_{1}+\frac{\delta t}{2} \nabla \cdot\left[\nabla \cdot \rho_{1} \mathbf{u u}\right] \\
\quad+(\tau-\delta t) \nabla \cdot\left[\frac{\rho_{1}}{\rho}\left(\nabla \rho c_{\mathrm{s}}^{2}-\mathbf{F}+\nabla \cdot \rho \mathbf{u u}+\partial_{t} \rho \mathbf{u}\right)\right] \\
\quad-c_{\mathrm{s}}^{2} \delta t \nabla \cdot\left(\beta \rho \frac{\rho_{1} \rho_{2}}{\rho^{2}} \hat{\mathbf{n}}\right)+(\tau-\delta t) \delta t \nabla \cdot\left[\frac{\rho_{1}}{\rho} \nabla \cdot \boldsymbol{\Psi}\right] .
\end{aligned}
$$

Writing $\rho_{1}=\rho \frac{\rho_{1}}{\rho}$ in the first line of the right-hand side of Eq. (31) and using the definition of actual macroscopic fluid velocity $\mathbf{v}$, Eq. (7), yields

$$
\begin{aligned}
\frac{\partial \rho_{1}}{\partial t} & +\nabla \cdot \rho_{1} \mathbf{v} \\
= & \frac{c_{\mathrm{s}}^{2} \delta t}{2} \nabla \cdot \rho \nabla \frac{\rho_{1}}{\rho}+\frac{\delta t}{2} \nabla \cdot\left[\nabla \frac{\rho_{1}}{\rho} \cdot \rho \mathbf{v v}\right] \\
& +\left(\tau-\frac{\delta t}{2}\right) \nabla \cdot\left[\frac{\rho_{1}}{\rho}\left(\nabla \rho c_{\mathrm{s}}^{2}-\mathbf{F}+\nabla \cdot \rho \mathbf{v v}+\partial_{t} \rho \mathbf{v}\right)\right] \\
& -c_{\mathrm{s}}^{2} \delta t \nabla \cdot\left(\beta \rho \frac{\rho_{1} \rho_{2}}{\rho^{2}} \hat{\mathbf{n}}\right)-\frac{\delta t^{2}}{2} \nabla \cdot\left[\nabla \cdot \frac{\rho_{1}}{\rho} \boldsymbol{\Psi}-\nabla \frac{\rho_{1}}{\rho} \cdot \boldsymbol{\Theta}\right] \\
& +(\tau-\delta t) \delta t \nabla \cdot\left[\frac{\rho_{1}}{\rho} \nabla \cdot \boldsymbol{\Theta}\right],
\end{aligned}
$$

where $\boldsymbol{\Theta}=\frac{\mathbf{F F} \delta t}{4 \rho}$ is used for brevity and time derivatives of $\mathbf{F}$ and $\mathbf{v}$ are assumed to be negligible compared to the spatial derivatives. A similar equation can be written for the second fluid as

$$
\begin{aligned}
\frac{\partial \rho_{2}}{\partial t}+ & \nabla \cdot \rho_{2} \mathbf{v} \\
= & \frac{c_{\mathrm{s}}^{2} \delta t}{2} \nabla \cdot \rho \boldsymbol{\nabla} \frac{\rho_{2}}{\rho}+\frac{\delta t}{2} \nabla \cdot\left[\nabla \frac{\rho_{2}}{\rho} \cdot \rho \mathbf{v} \mathbf{v}\right] \\
& +\left(\tau-\frac{\delta t}{2}\right) \nabla \cdot\left[\frac{\rho_{2}}{\rho}\left(\nabla \rho c_{\mathrm{s}}^{2}-\mathbf{F}+\nabla \cdot \rho \mathbf{v v}+\partial_{t} \rho \mathbf{v}\right)\right] \\
& +c_{\mathrm{s}}^{2} \delta t \nabla \cdot\left(\beta \rho \frac{\rho_{1} \rho_{2}}{\rho^{2}} \hat{\mathbf{n}}\right)-\frac{\delta t^{2}}{2} \nabla \cdot\left[\nabla \cdot \frac{\rho_{2}}{\rho} \boldsymbol{\Psi}-\nabla \frac{\rho_{2}}{\rho} \cdot \boldsymbol{\Theta}\right] \\
& +(\tau-\delta t) \delta t \nabla \cdot\left[\frac{\rho_{2}}{\rho} \nabla \cdot \boldsymbol{\Theta}\right],
\end{aligned}
$$

where the sign of the segregation term is corrected for the second fluid. From consideration of the local mass conservation of the combined two phases, one expects that the continuity equation should be recovered. For the combined two-phase system, addition of Eq. (32) and Eq. (33) gives

$$
\begin{aligned}
\frac{\partial \rho}{\partial t}+ & \nabla \cdot \rho \mathbf{v} \\
= & -\frac{\delta t^{2}}{2} \nabla \cdot \nabla \cdot \Psi+(\tau-\delta t) \delta t \nabla \cdot \nabla \cdot \boldsymbol{\Theta} \\
& +\left[\tau-\frac{\delta t}{2}\right] \nabla \cdot\left[\nabla \rho c_{\mathrm{s}}^{2}-\mathbf{F}+\nabla \cdot \rho \mathbf{v} \mathbf{v}+\partial_{t} \rho \mathbf{v}\right] .
\end{aligned}
$$


From the Chapman-Enskog analysis [27] one remembers that in the first order of the Knudsen number (the effective smallness parameter) approximation, the differential operators are expanded only up to the first order in space. For this reason, the Eulerian terms on the right-hand side of Eq. (34) are usually neglected $[33,35]$. The interfacial force term is of the order of fluid velocity in multiphase situations, $\mathbf{F} \sim \delta t \frac{\mathbf{v}}{\rho}$. The first line on the right-hand side of Eq. (34) is effectively of the second order in fluid velocity and is neglected. With this simplification, we arrive at the usual continuity equation $\frac{\partial \rho}{\partial t}+\nabla \cdot \rho \mathbf{v}=0$. The dynamics of the phase field $\varphi$, defined in Eq. (3) can be found by looking at the difference of continuity equations of the two fluids. Within the low Mach number assumption ( $\left.\mathrm{Ma}=\frac{|\mathbf{v}|}{c_{\mathrm{s}}} \ll 1\right)$ of the weakly compressible limit, the change of total density across the interface, $\Delta \rho$, is assumed to be negligible compared to the total density of the fluids, $\frac{\Delta \rho}{\rho} \ll 1$. This assumption holds true even in the presence of external forces like gravity or surface tension. Subtraction of Eq. (33) from Eq. (32), and using Eq. (3), yields

$$
\begin{aligned}
\frac{\partial \varphi}{\partial t}+ & \nabla \cdot \varphi \mathbf{v} \\
= & \frac{c_{\mathrm{s}}^{2} \delta t}{2 \rho} \nabla \cdot \rho\left[\nabla \varphi-\beta\left(1-\varphi^{2}\right) \hat{\mathbf{n}}\right] \\
& +\frac{1}{\rho}\left(\tau-\frac{\delta t}{2}\right) \nabla \cdot\left[\varphi\left(\nabla \rho c_{\mathrm{s}}^{2}-\mathbf{F}+\nabla \cdot \rho \mathbf{v v}+\partial_{t} \rho \mathbf{v}\right)\right] \\
& +\frac{\delta t}{2 \rho} \nabla \cdot[\nabla \varphi \cdot(\rho \mathbf{v} \mathbf{v}+\delta t \boldsymbol{\Theta})-\delta t \nabla \cdot \varphi \mathbf{\Psi}] \\
& +(\tau-\delta t) \frac{\delta t}{\rho} \nabla \cdot[\varphi \nabla \cdot \boldsymbol{\Theta}] .
\end{aligned}
$$

It is instructive to compare the phase field dynamics obtained in one of the previous studies [18,23] that employed Chapman-Enskog analysis and included the surface tension forces with Guo's forcing scheme [36]. In the present notation, Eq. (21) in the study by Hollis et al. [18] stands as

$$
\begin{aligned}
\frac{\partial \varphi}{\partial t}+\nabla \cdot \varphi \mathbf{v}= & \frac{1}{2 \rho} \nabla \cdot\left[\varphi\left(\nabla \rho c_{\mathrm{s}}^{2}-\mathbf{F}\right)\right] \\
& +\frac{\delta t}{2 \rho} \nabla \cdot[\nabla \cdot(\rho \varphi \mathbf{v} \mathbf{v})+\delta t \nabla \cdot \varphi \mathbf{\Psi}] .
\end{aligned}
$$

A numerical kinematic condition for the fluid interface was suggested in their study [18], where the local fluid viscosity is increased inside the interface, to ensure $\frac{\partial \varphi}{\partial t}+\nabla \cdot \varphi \mathbf{v}=0$. A comparison of Eq. (36) and Eq. (35) reveals the differences in the coefficients of the terms that are second order in fluid velocity and the shear stress terms. More importantly, the present method correctly recovers the terms [the first line on the right-hand side of Eq. (35)] responsible for maintaining a finite interface width. Note that the surface tension forces [see Eq. (8)] do not explicitly assume any shape of the phase field in the CG models. The interface width, therefore, must be apparent in some form in the macroscopic description of the fluid dynamics, in this case in the continuity equations of the individual fluids. More recently, Burgin et al. [20] analyzed evolution of the individual density fields as a result of the segregation operation for the case of fluids with a density contrast. The emergent behavior of the first density field obtained in their study, applied to the equal density case and in the present notation, is

$$
\begin{aligned}
& \frac{\partial \rho_{1}}{\partial t}+\frac{\delta t}{2} \frac{\partial^{2} \rho_{1}}{\partial t^{2}}+\nabla \cdot \rho_{1} \mathbf{v} \\
& =\frac{c_{\mathrm{s}}^{2} \delta t}{2} \nabla^{2} \rho_{1}+\frac{\delta t}{2} \nabla \cdot\left[\nabla \cdot \rho_{1} \mathbf{v v}\right]-c_{\mathrm{s}}^{2} \delta t \nabla \cdot\left(\beta \rho \frac{\rho_{1} \rho_{2}}{\rho^{2}} \hat{\mathbf{n}}\right) .
\end{aligned}
$$

Equation (37) (and another one for the density of the second fluid) was derived using a procedure similar to the present work, i.e., by considering local changes in the fluid density. The difference being that they expanded the temporal derivatives up to the second order. In addition, Eq. (37) was derived for a planar interface under the assumption of negligible fluid velocity gradients. Burgin et al. [20] utilized these density evolution equations further to discuss the correction terms for the density contrast case via the Chapman-Enskog expansion. Under the assumption of negligible velocity gradients only the equilibrium fluid populations are important. A comparison of Eq. (37) with Eq. (23) and Eq. (30) shows excellent agreement, besides the term containing a second-order time derivative of density of the first fluid $\rho_{1}$. Here, in the form of Eq. (31), we extend Eq. (37) to an arbitrarily shaped interface while taking into account the effect of nonequilibrium part of the fluid populations.

In the present study, we focus upon the terms on the righthand side of Eq. (35), which ensure constant interface width. We neglect the second order in velocity and Eulerian terms in Eq. (35), to arrive at

$$
\frac{\partial \varphi}{\partial t}+\nabla \cdot \varphi \mathbf{v}=\frac{c_{\mathrm{s}}^{2} \delta t}{2}\left(\nabla^{2} \varphi-\beta \nabla \cdot\left[1-\varphi^{2}\right] \hat{\mathbf{n}}\right),
$$

where $M=\frac{c_{\mathrm{s}}^{2} \delta t}{2}$ is the mobility. Equation (38) is an AllenCahn equation, first proposed by Sun and Beckermann [37] for diffuse interface tracking and later adopted in a conservative form $[21,38,39]$. It is noteworthy that the original segregation operator [30] (naturally written in a conservative form) which leads to Eq. (38) was proposed at least a decade earlier than the method of Sun and Beckermann [37]. It is seen that the only physical quantity entering in the phase field evolution equation is the fluid velocity $\mathbf{v}$, which results from the solution of the fluid dynamical equations. The surface tension and other external forces, therefore, influence the interface motion via the fluid velocity only. At steady state, the equilibrium profile for the phase field variable for a radially symmetric symmetric geometry can be derived as

$$
\varphi^{\mathrm{eq}}(r)= \pm \tanh \left[\beta\left(r-r_{\mathrm{I}}\right)\right]
$$

where $r$ is a variable measured along a direction normal to the interface and $r_{\mathrm{I}}$ shows the location of the sharp interface. Equation (38), by construction, does not have a contribution from the interface curvature. This situation is different compared to the one occurring in the phase transition problems, where a curvature induced capillary force is one of the factors driving the interface motion. To see this, we write the righthand side of Eq. (38) in orthogonal curvilinear coordinates with unit vectors $\hat{\mathbf{r}}$ and $\hat{\mathbf{s}}$ that are in a direction perpendicular and tangential to the interface, respectively. Using a 
transformation rule for spatial derivatives in the curvilinear coordinates [40], and assuming only a small deviation from equilibrium, the right-hand side (R.H.S.) of Eq. (38) is then written as

$$
\begin{aligned}
\text { R.H.S. }= & \frac{c_{\mathrm{s}}^{2} \delta t}{2}\left[\frac{\partial^{2}}{\partial r^{2}}-\frac{\kappa}{1-r \kappa} \frac{\partial}{\partial r}+\frac{1}{(1-r \kappa)^{2}} \frac{\partial^{2}}{\partial s^{2}}\right] \varphi \\
& +\frac{c_{\mathrm{s}}^{2} \delta t}{2} \frac{r}{(1-r \kappa)^{3}} \frac{\partial \kappa}{\partial s} \frac{\partial}{\partial s} \varphi \\
& -\frac{c_{\mathrm{s}}^{2} \delta t}{2}\left[\frac{\partial}{\partial r}-\frac{\kappa}{1-r \kappa}\right] \beta\left(1-\varphi^{2}\right) \\
= & \frac{c_{\mathrm{s}}^{2} \delta t}{2}\left(\frac{\partial}{\partial r}-\frac{\kappa}{1-r \kappa}\right)\left(\frac{\partial}{\partial r}+2 \beta \varphi^{\mathrm{eq}}\right) \varphi^{\text {neq }} \\
& +\frac{c_{\mathrm{s}}^{2} \delta t}{2(1-r \kappa)^{2}}\left[\frac{\partial^{2}}{\partial s^{2}} \varphi^{\mathrm{neq}}+\frac{r}{(1-r \kappa)} \frac{\partial \kappa}{\partial s} \frac{\partial}{\partial s} \varphi^{\mathrm{neq}}\right],
\end{aligned}
$$

where the radial distance $r$ is such that $|r \kappa| \ll 1$, the variable $s$ measures distance along the tangential direction $\hat{\mathbf{s}}$ and $\varphi^{\text {neq }}$ is the deviation of $\varphi$ from its equilibrium profile. Close to the equilibrium solution $\varphi(r, s)=\varphi^{\mathrm{eq}}(r)+\varphi^{\text {neq }}(r, s)$ with $\frac{\partial \varphi^{\mathrm{eq}}}{\partial r}=$ $\beta\left(1-\varphi^{2}\right) \hat{\mathbf{n}}$.

To judge the relative importance of the terms in Eq. (40), we define a dimensionless stretched coordinate $\eta=r \beta$ that measures the normal distance from the interface. This coordinate $\eta$ reminds us that the phase field variable $\varphi$ changes more rapidly along the normal direction when compared to the tangential one. For a given physical length $L$, the limit of sufficiently small interface width then corresponds to $\beta \gg \frac{1}{L}$. In other words, the terms in Eq. (40) become more important with increasing exponent of $\beta$. Using additional information that the radial distance $r$ of interest is much smaller than the radius of curvature, i.e., $(1-r \kappa)^{n} \approx 1-n r \kappa$, Eq. (40) now reads

\section{R.H.S}

$$
\begin{aligned}
= & \frac{\beta c_{\mathrm{s}}^{2} \delta t}{2}\left(\beta \frac{\partial}{\partial \eta}-\kappa\left[1+\frac{\kappa \eta}{\beta}\right]\right)\left(\frac{\partial}{\partial \eta}+2 \varphi^{\mathrm{eq}}\right) \varphi^{\mathrm{neq}} \\
& +\frac{c_{\mathrm{s}}^{2} \delta t}{2}\left[1+2 \frac{\kappa \eta}{\beta}\right]\left(\frac{\partial^{2}}{\partial s^{2}} \varphi^{\mathrm{neq}}+\frac{\eta}{\beta}\left[1+\frac{\kappa \eta}{\beta}\right] \frac{\partial \kappa}{\partial s} \frac{\partial}{\partial s} \varphi^{\mathrm{neq}}\right) .
\end{aligned}
$$

The terms containing partial derivatives with respect to the tangential coordinate $s$ on the second line of Eq. (41) are responsible for a mechanism that restores the radial symmetry. Among these terms, $\frac{c_{s}^{2} \delta t}{2} \frac{\partial^{2}}{\partial s^{2}} \varphi^{\text {neq }}$ dominates the remaining ones, having the highest exponent of $\beta$. Thus, in addition to advection via fluid velocity, there is an artificial surface diffusion-like mechanism that attempts to achieve a radial symmetry. This result has the important consequence that the arbitrarily shaped interface between two fluids will finally converge to a radially symmetric distribution even when the surface tension between the fluids is zero. In the usual flow situations, advection due to fluid velocity dominates the diffusive transport along the tangential direction of the interface and thus ensures a correct description of the continuity equation for each of the fluids.
Any numerical approach dealing with multiphase fluid system aims to prescribe a purely advective equation for the phase field variable, or alternatively, for the individual fluid density fields. The numerical solution of such a purely advection equation poses its own difficulties, while a diffusionadvection equation of the form Eq. (38) alleviates these difficulties in addition to ensuring a finite interface width. Other numerical multiphase fluid methods, that explicitly solve Eq. $(38)[21,22]$ or an equation similar in spirit like the level set method [41] for interface tracking, tune the mobility of the phase field to minimize the effect of diffusion along the tangential direction of the interface. A comparison of Eq. (38) with the one used by Geier et al. [21] shows that the phase field mobility $M$ is no longer a free parameter in the CG model and is equal to $\frac{c_{\delta}^{2} \delta t}{2}$ for the equal reference density case. The parameter $\beta$ appearing in Eq. (9) and Eq. (10) is chosen to be $\beta=\frac{2}{W}$, where $W$ is the interface width.

Similar to the single-relaxation-time BGK [28] collision operator, the more sophisticated two-relaxation-time (TRT) [42] and MRT [43] collision operators expand the fluid populations $N_{i}$ around the equilibrium fluid populations $N_{i}^{\text {eq }}$. From Eq. (23) and Eq. (30), it is clear that the phase field mobility is determined by the form of equilibrium fluid populations alone. Thus, the phase field mobility $M$ is expected to remain the same when the TRT or MRT collision operators are employed.

\section{NUMERICAL RESULTS}

The purpose of the numerical simulations in this section is twofold. The first one is to establish that the phase field evolution equation which is indirectly solved in the CG model is given by Eq. (38). It remains important to show that the interface width corresponds to the one predicted by Eq. (38) and the phase field mobility is indeed $\frac{c_{s}^{2} \delta t}{2}$ and independent of the viscosities of the fluids. Further, in the absence of surface tension, an arbitrarily shaped fluid interface should eventually attain a radially symmetric profile as suggested by Eq. (41). The second purpose is to show that the segregation operator II, Eq. (10), being isotropic in the leading-order error of spatial discretization, indeed helps to reduce the spurious currents when compared to the original segregation operator, Eq. (9).

\section{A. Kinetics of the interface motion}

A standard way to test the ability of an advection scheme is to simulate the Zalesak's disk problem [25]. A test of the three-phase extension of the original CG method using this benchmark has been performed [14]. The focus of that work [14], however, was directed towards analyzing the shape of triple-phase junctions and the effect of Mach number and viscosity ratio upon them. Here our interest lies in establishing the fact that kinetics of the resulting phase field equation in CG model depends solely upon the lattice speed of sound and is independent of the viscosity and surface tension of the fluids.

Figure 2 depicts the initial configuration of the system geometry. All the quantities reported below are given in lattice units (lu). A slotted disk with a radius $R=80$ and a slot cut from center with width $d=15$ is filled with the first fluid 


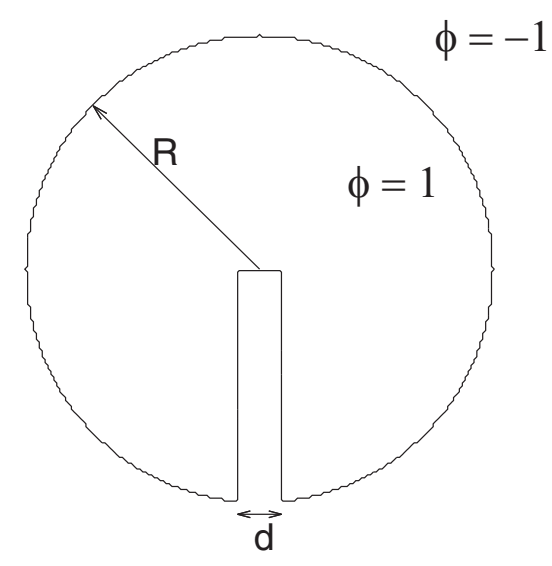

FIG. 2. Initial configuration of the two-phase system for Zalesak's disk problem. The slotted disk has a radius $R$ of 80 with a slot cut from the center with a width $d$ of 15 . The interior of the disk is filled with the first fluid (with $\varphi=1$ ) and the exterior with the second fluid (with $\varphi=-1$ ). The interface between the fluids is shown with the isocontour corresponding to $\varphi=0.0$.

while the rest of the system consists of the second fluid. The densities and kinematic viscosities of both fluids are assumed to be equal and are set to 1 and 0.16 , respectively. The system is initiated with a temporally constant and spatially varying velocity field $\mathbf{u}^{\text {forced }}(\mathbf{x})=u_{0} \pi\left(\frac{y}{L}-\frac{1}{2},-\frac{x}{L}+\frac{1}{2}\right)$ with $L=200$ being the system size and $u_{0}=0.02$ the strength of the velocity field. At each time step the fluid velocity is set to $\mathbf{u}^{\text {forced }}(\mathbf{x})$, and the corresponding equilibrium fluid populations [see Eq. (2)] are calculated. The disk completes one rotation around itself in time $T_{\text {rot }}=\frac{2 L}{u_{0}}=20000$. The interface width $W$ is selected to be 3 .

The flow Péclet number Pé $=\frac{L W}{M}$ measures the relative importance of the advective and diffusive transports. For the present model, phase field mobility $M=\frac{c_{\delta}^{2} \delta t}{2}$, yielding Pé $=$ 0.36. Another important dimensionless number in the current context is the Cahn number defined as $\mathrm{Ca}=\frac{W}{L}=0.015$ that signifies how large the auxiliary interface width is when compared to the physical length scale of the system. A large value of $W$ ensures that the spatial derivatives are evaluated accurately. However, increasing the value of $\mathrm{W}$ also makes it necessary to increase the system size thus increasing the computational cost. In practice, an optimal value of $W$ is usually chosen such that a reasonable accuracy is obtained within a given computational cost constraint.

Figure 3(a) compares the state of the disk after one full revolution (dashed line) to the original state. Given the large phase field mobility of the CG method, the sharp corners of the disk can be seen to be smeared out. Nevertheless, the overall shape of the disk after one revolution agrees well with the initial state of the disk.

Next, we solve Eq. (38) directly along the lines outlined by Geier et al. [21]. A comparison of the disks, solved using Eq. (38) and the CG model, after one complete revolution is shown in Fig. 3(b). It is seen from the figure that solutions obtained via both the numerical schemes indeed lie on top of each other. This excellent agreement between the two numerical methods justifies neglecting the terms that appear in the third order of spatial discretization and second order in fluid velocity. The sensitivity of the phase field mobility $M$ can be seen in the same subfigure, where a nearly two orders of magnitude reduction $M$ results in a better approximation of the initial disk profile. This case of $M=0.001$ can be seen as a representation of the phase field advection Eq. (36). To further establish the fact that the phase field mobility $M$ is independent of the fluid viscosities, the same Fig. 3(c) shows that changing the fluid viscosities from 1.0 to 0.01 results in the same final disk profile. It is to be noted that the smeared nature of the corners, visible from numerical solution of $\mathrm{CG}$ model, is a result of the nonzero phase field mobility and not due to surface tension induced capillary effects. Figure 3(d) further illustrates this result, where the surface tension is varied by two orders of magnitude and essentially the same final phase field profile is obtained.

To demonstrate the effect of a diffusion term on the interface evolution, we investigate the case where surface tension is zero. Figure 4 shows the initial configuration of the fluid distribution with the first fluid is enclosed in a square box (size $l=20$ ) inside the second fluid. The system size $L$ is 100 , the interface width is $W=3$, and the kinematic viscosity of both the fluids is 0.16 . In the absence of surface tension and any fluid velocity, the fluid interface should retain its shape apart from broadening of the interface due to the diffuse interface construction of the CG method. However, in (a)

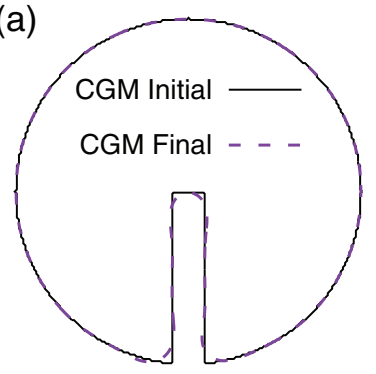

(b)

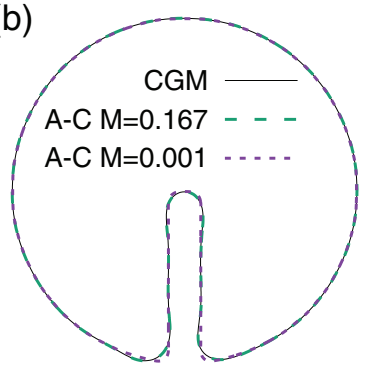

(c)

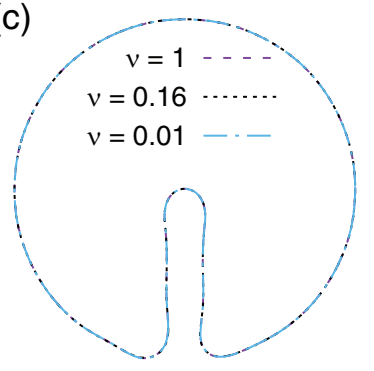

(d)

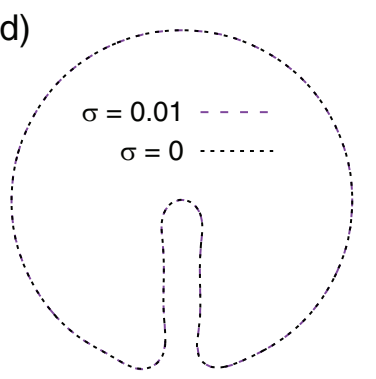

FIG. 3. (a) A comparison of the initial state and the state after one rotation using the CG method for flow Péclet number Pé $=0.36$. The sharp edges of initial interface profile are smeared out. (b) Direct numerical solution of Allen-Cahn Eq. (38) agrees well with the CG solution when the mobility of the Allen-Cahn equation is adjusted such that it matches with the CG mobility $\left(M=\frac{c_{\mathrm{S}}^{2} \delta t}{2}\right)$. For a lower mobility of $M=0.001$, the smearing of corners is reduced. This mobility $M=0.001$ approximates well the advection phase field Eq. (36) when the right-hand side is negligible. (c) A comparison of interface position resulting from CG model for different viscosities. Surface tension $\sigma=0$ in all of these cases. (d) A similar comparison for two different surface tension values with kinematic viscosity $v=0.16$. 

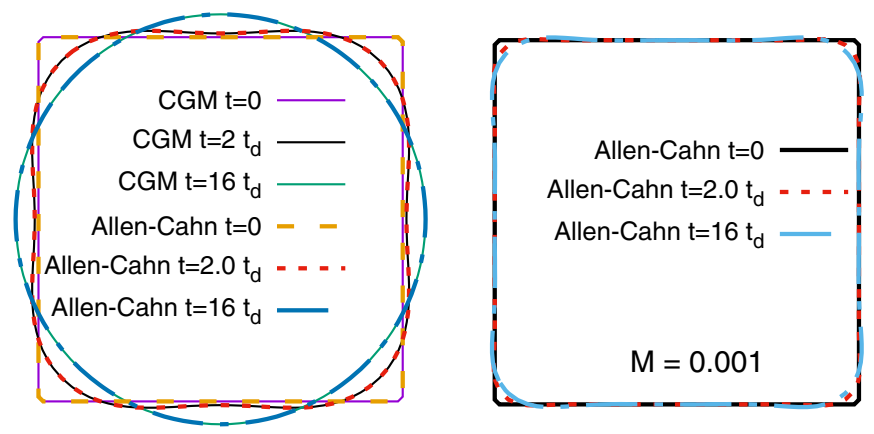

FIG. 4. (a) Evolution of the fluid-fluid interface when the surface tension is zero in the CG model. Initially, the first fluid in the shape of a square is surrounded by the second fluid. The interface profile here is shown for three times $t=0,2 t_{\mathrm{d}}$, and $8 t_{\mathrm{d}}$ with solid lines for the CG model. The solution obtained via the Allen-Cahn equation, Eq. (38), with the same interface width $W$ and the mobility $M=\frac{c_{\mathrm{S}}^{2} \delta t}{2}$ are shown with dashed lines. (b) Interface evolution with the Allen-Cahn Eq. (38) with a low mobility $M=0.001$. The interface becomes smooth near the sharp corners of the initial square in a relatively short amount of time. Given a nearly 167 times lower $M$, the surface diffusion time $t_{\mathrm{d}}$ is as much higher and the interface evolves towards a radial symmetry much slower than with the case of $M=\frac{c_{\mathrm{s}}^{2} \delta t}{2}$.

this case, the initial interface shape does not have a radial symmetry, and therefore the term $\frac{c_{\mathrm{S}}^{2} \delta t}{2} \frac{\partial^{2}}{\partial s^{2}} \varphi^{\text {neq }}$ in Eq. (41) becomes an important factor to drive the interface motion. The timescale at which this diffusion mechanism alters the shape of the interface can be estimated as $t_{\mathrm{d}}=\frac{l^{2}}{M}=\frac{2 l^{2}}{c_{\mathrm{S}}^{2} \delta t}$ for a fluid body of typical length $l$. Thus, the lower the value of the phase field mobility $M$ the higher is the typical interface diffusion time. Decreasing the value of $M$ arbitrarily, however, gives rise to numerical errors [38], while too high of a value produces unphysical interface motion. Physically meaningful results can be obtained with the $\mathrm{CG}$ model as long as the interface diffusion time $t_{\mathrm{d}} \gg t_{\mathrm{a}}$, where $t_{\mathrm{a}}=\frac{l}{|\mathbf{v}|}$ is the advective timescale of the system.

The evolution of the interface in the CG model, as depicted in Fig. 4(a) with solid lines, sheds light on the effect of diffusion mechanism active along the tangential direction of the interface. The steady-state interface profile, in this case, is indeed a circular shape. Figure 4(a) also shows the numerical solution obtained with Eq. (38) with dashed lines for the same physical times $t=0,2 t_{\mathrm{d}}$ and $8 t_{\mathrm{d}}$. The interface width and mobility of the phase field are chosen to match the CG model. The excellent agreement of the solutions obtained with Allen-Cahn equation Eq. (38) and the CG model confirms the prediction of phase field mobility in the present work. For more clarity, Fig. 4(b) shows the solution obtained with Allen-Cahn Eq. (38) with a smaller mobility $M=0.001$. In this case, the strength of the interface diffusion along the tangential direction is reduced dramatically, and the phase field profile changes only slightly within the time $t=8 t_{\mathrm{d}}$ where $t_{\mathrm{d}}$ is evaluated for mobility $M=\frac{c_{\mathrm{s}}^{2} \delta t}{2}$.

\section{B. Spurious currents}

Spurious currents are an unphysical fluid velocity field near curved interfacial area due to the discretization errors. Two-
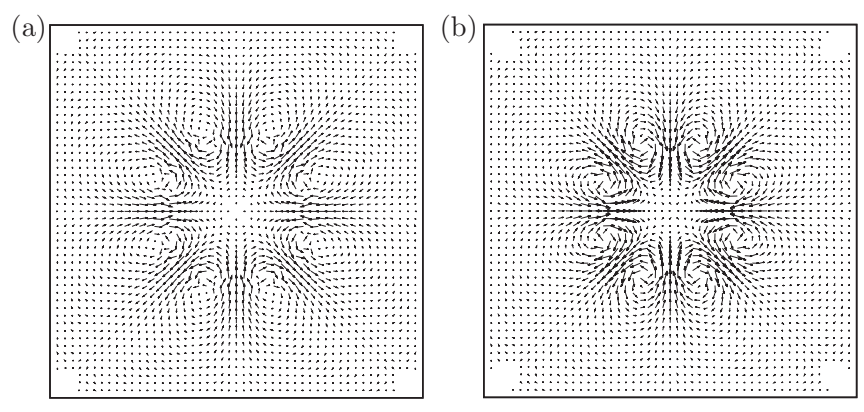

FIG. 5. A comparison of the spurious currents for the segregation (a) operator I and (b) operator II [see Eq. (9) and Eq. (10)] at fixed value of surface tension $\sigma=2 \times 10^{-2}$ for a steady circular drop. The spurious currents are scaled with the same factor $\left(=8 \times 10^{5}\right)$ for visualization purpose. It is seen that the use of Eq. (10) indeed reduces the magnitude of the spurious currents.

phase interaction terms, within LB frameworks, are usually computed with finite difference stencils. A fairly straightforward but computationally expensive way to control the discretization errors is to use higher-order isotropic stencils or increase the grid resolution [31,44]. As demonstrated by Pooley and Furtado [45] for the free energy LB method, in some cases, the finite difference stencils can be chosen such that the discretization errors can be significantly reduced without resorting to higher-order stencils. Here we aim to numerically investigate the effect of discretization errors due to the segregation operators given by Eq. (9) and Eq. (10). For this purpose, a circular drop in two dimensions is investigated for various values of the surface tension $\sigma$. The kinematic viscosity of both fluids $v$ is set to 0.16 , the system size $L$ is 100 , and the radius of the drop is 20 .

To make a consistent comparison between these two segregation operators, the interface width must be the same for both the cases. From Eq. (28) and Eq. (30), we see that the interface thickness controlling parameter $\beta$ is related by

$$
\beta^{\mathrm{II}}=\frac{4+\sqrt{2}}{6} \beta^{\mathrm{I}},
$$

where the superscript denotes the segregation operator used. Thus, the interface width, which is inversely proportional to $\beta$, is always larger for segregation operator II. We have used $\beta^{I}=0.7$ and $\beta^{I I}$ as given by Eq. (42).

Figure 5 shows the comparison of the velocity fields obtained for a fixed value of $\sigma=2 \times 10^{-2}$ using Eq. (9) and Eq. (10), respectively. The arrow size gives the magnitude of the spurious currents, while their direction aligns with the velocity field. At the leading order of truncation, Eq. (9) generates anisotropic spatial derivatives [see Eq. (A3)] that depend upon the number of spatial dimensions. These spatially anisotropic terms then act as one of the sources of spurious currents. This effect is clearly seen in Fig. 5. It is of interest to know how the segregation operator II performs at different values of the surface tension $\sigma$ and relaxation parameter $\tau$. Figures 6 and 7 show the magnitude of the maximum spurious currents for varying surface tension and relaxation parameter values, respectively. The segregation operator II produces less spurious currents than operator I for both of these cases. 


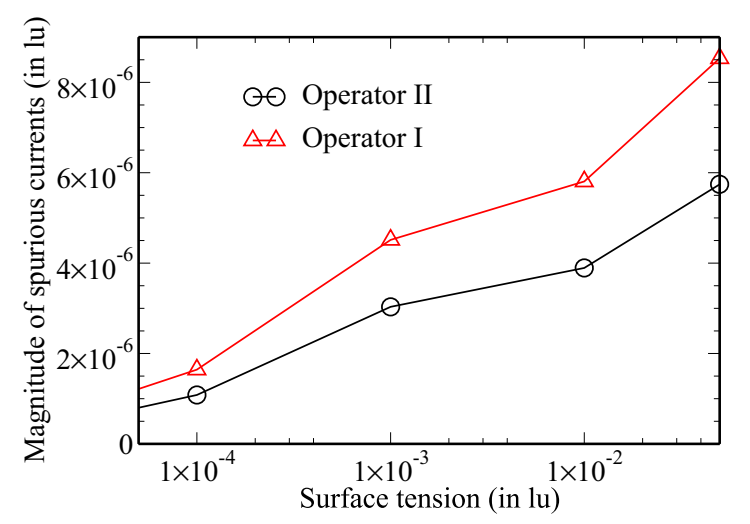

FIG. 6. Dependence of the magnitude of spurious currents upon surface tension for the two segregation schemes, Eq. (9) and Eq. (10). The improved segregation scheme reduces the magnitude of the spurious currents for a range of surface tension values when compared with the original one.

The TRT or MRT collision operators allow us to choose the bulk viscosity of the fluid by tuning the kinetic relaxation parameters $[42,43]$. The choice of these relaxation parameters can, via the bulk viscosity, influence the residual or the spurious velocity fields at the steady state. Therefore, the segregation operator II is expected to perform better than the operator I even with TRT or MRT collision operators, although overall less spurious currents will be produced in both cases as compared to the SRT collision operator.

\section{CONCLUSION}

In this work, we analyze the phase field evolution equation in the CG model for an equal density case of two-phase fluids. A systematic application of the Taylor series expansion to LB equation of the two fluid phases leads to the phase field equation of the interface. This equation is shown to exhibit a conservative Allen-Cahn-type nature. Importantly,

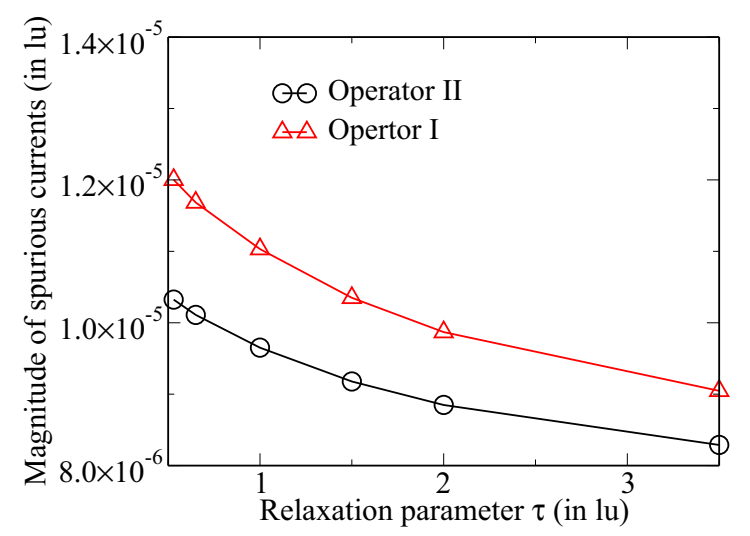

FIG. 7. Dependence of the magnitude of spurious currents upon relaxation time $\tau$ for the two segregation schemes, Eq. (9) and Eq. (10). The surface tension $\sigma$ is fixed at $1 \times 10^{-3}$. The magnitude of the spurious currents decreases for both of the segregation operators for increasing $\tau$. the mobility of the phase field equation, in this case, does not depend upon the physical properties of the fluids like viscosity or surface tension. The nearly constant phase field mobility is verified by comparison of the CG model and direct solution of the phase field equation in Zalesak's disk test case. The case of unequal densities of the two fluids is handled by changing the rest lattice weights $w_{0}$ or assigning different lattice speed of sound for the fluids in the CG model $[10,46]$. Such a change of lattice speed of sound violates the momentum conservation equation, and additional correction terms are necessary. Thus, in the context of the present analysis, the phase field mobility in this case will be determined by the effective lattice speed of sound between the fluids as shown by Burgin et al. [20].

A consideration of the density exchange via Taylor expansion gives a direct access to spatial discretization terms involved in the resulting continuity equation of the fluids. This analysis reveals that the original segregation operator [6], Eq. (9), produces a spatial discretization error that is anisotropic in the third order of expansion. Given that these error terms contribute to undesirable spurious currents in the interfacial region, we also study a slightly modified segregation operator by Halliday et al. [23]. As expected, this modified segregation operator is shown to produce less spurious currents compared to the original one for all values of surface tensions. This result highlights the fact that, in addition to the isotropy of the imposed surface tension force (or the perturbation operator), isotropy of the segregation also plays an important role in reducing the spurious currents.

\section{ACKNOWLEDGMENTS}

The authors acknowledge the support from the Bundesministerium für Bildung und Forschung under the project MERID and the Helmholtz Association within the program Renewable Energies, 35.14.

\section{APPENDIX: EXCHANGE TERMS APPEARING IN PHASE FIELD EQUATION}

Let the spatially dependent term be denoted by $T_{i}(\mathbf{x})$. Then the net change in the density of the first fluid $\delta \rho_{1}$ at any node $\mathbf{x}$ due to this term is $\sum_{i}\left[T_{i}\left(\mathbf{x}-\mathbf{c}_{i} \delta t\right)-T_{i}(\mathbf{x})\right]$. We expand $T_{i}\left(\mathbf{x}-\mathbf{c}_{i} \delta t\right)$ as a Taylor series up to the third order in space around the node $\mathbf{x}$ to arrive at the final expression for different forms of $T_{i}(\mathbf{x})$ in the following:

(1) $T_{i}=w_{i} \chi$ for a scalar $\chi$ :

$$
\delta \rho_{1}=\frac{c_{\mathrm{s}}^{2} \delta t^{2}}{2} \nabla^{2} \chi
$$

Note that this expression is exact up to the third order in space, and the leading-order isotropic error term behaves as $O\left(\nabla^{4} \psi\right)$ [31,47].

(2) $T_{i}=w_{i} \frac{\boldsymbol{\psi} \cdot \mathbf{c}_{i}}{c_{\mathrm{s}}^{2}}$ for a vector $\boldsymbol{\psi}$ :

$$
\delta \rho_{1}=-\delta t\left(1+\delta t^{2} \frac{c_{\mathrm{s}}^{2}}{2} \nabla^{2}\right) \nabla \cdot \boldsymbol{\psi} .
$$


(3) $T_{i}=w_{i}^{\text {aniso }} \frac{\boldsymbol{\psi} \cdot \mathrm{c}_{i}}{c_{\mathrm{S}}^{2}}$ for a vector $\boldsymbol{\psi}$ :

$$
\begin{aligned}
\delta \rho_{1}= & -\frac{4+\sqrt{2}}{6} \delta t \nabla \cdot \boldsymbol{\psi}-\frac{4+\sqrt{2}}{36} \delta t^{3}\left(\frac{\partial^{3} \psi_{x}}{\partial x^{3}}+\frac{\partial^{3} \psi_{y}}{\partial y^{3}}\right) \\
& -\frac{3 \sqrt{2}}{36} \delta t^{3}\left(\frac{\partial}{\partial x} \frac{\partial^{2} \psi_{x}}{\partial y^{2}}+\frac{\partial}{\partial y} \frac{\partial^{2} \psi_{y}}{\partial x^{2}}\right),
\end{aligned}
$$

where $\psi_{x}$ and $\psi_{y}$ are the components of the vector $\boldsymbol{\psi}$.

(4) $T_{i}=w_{i} \frac{\left(\boldsymbol{\psi} \cdot \mathbf{c}_{i}\right)\left(\xi \cdot \mathbf{c}_{i}\right)}{c_{\mathrm{s}}^{4}}$ for vectors $\boldsymbol{\psi}$ and $\boldsymbol{\xi}$.

$$
\delta \rho_{1}=\delta t^{2} \frac{\nabla^{2}(\boldsymbol{\psi} \cdot \xi)}{2}+\frac{\delta t^{2}}{2} \nabla \cdot \nabla \cdot[\psi \xi \xi \xi \psi] .
$$

(5) $T_{i}=w_{i} \chi \mathbf{c}_{i} \cdot \nabla \zeta$ for scalars $\chi$ and $\zeta$ :

$$
\delta \rho_{1}=-\delta t^{2} c_{\mathrm{s}}^{2}\left(\nabla \chi \cdot \nabla \zeta+\chi \nabla^{2} \zeta\right) .
$$

(6) $T_{i}=\frac{w_{i}}{c_{\mathrm{S}}^{2}} \chi \mathbf{c}_{i} \cdot \nabla\left(\boldsymbol{\psi} \cdot \mathbf{c}_{i}\right)$ for a scalar $\chi$ and a vector $\boldsymbol{\psi}$ :

$$
\begin{aligned}
\delta \rho_{1}= & -\frac{\delta t^{2}}{3} \boldsymbol{\psi} \cdot \nabla \nabla^{2} \chi+\frac{\delta t^{2}}{3} \nabla \chi \cdot \nabla(\nabla \cdot \psi) \\
& +\frac{\delta t^{2}}{6} \nabla \cdot \nabla \cdot[(\boldsymbol{\psi} \nabla \chi)+\nabla \chi \psi] \\
& -\frac{\delta t^{2}}{6} \nabla \cdot \psi \nabla^{2} \chi+\frac{\delta t^{2}}{2} \chi \nabla^{2} \nabla \cdot \psi+\frac{\delta t^{2}}{3} \nabla \chi \cdot \nabla^{2} \psi .
\end{aligned}
$$

(7) $T=w_{i} \chi \mathbf{c}_{i} \cdot \nabla\left(\frac{\boldsymbol{\psi} \cdot \mathbf{c}_{\mathbf{i}} \cdot \mathbf{c}_{i}}{2 c_{\mathrm{s}}^{4}}-\frac{\boldsymbol{\psi} \cdot \boldsymbol{\xi}}{2 c_{\mathrm{s}}^{2}}\right)$ for a scalar $\chi$ and vectors $\psi$ and $\xi$ :

$$
\delta \rho_{1}=\frac{\delta t^{2}}{2} \nabla \cdot(\chi[\nabla \cdot(\xi \psi+\psi \xi)]) .
$$

Note that the above expressions [except for Eq. (A3)] are equally valid for all rectangular lattices like D2Q9, D3Q19, or D3Q27.
[1] X. Shan and H. Chen, Phys. Rev. E 47, 1815 (1993).

[2] A. K. Gunstensen, D. H. Rothman, S. Zaleski, and G. Zanetti, Phys. Rev. A 43, 4320 (1991).

[3] M. R. Swift, W. R. Osborn, and J. M. Yeomans, Phys. Rev. Lett. 75, 830 (1995).

[4] X. He, S. Chen, and R. Zhang, J. Comput. Phys. 152, 642 (1999).

[5] H. Huang, M. Sukop, and X. Lu, Multiphase Lattice Boltzmann Methods: Theory and Application (John Wiley \& Sons, New York, 2015).

[6] M. Latva-Kokko and D. H. Rothman, Phys. Rev. E 71, 056702 (2005).

[7] T. Reis and T. N. Phillips, J. Phys. A 40, 4033 (2007).

[8] H. Huang, J.-J. Huang, X.-Y. Lu, and M. C. Sukop, Int. J. Mod. Phys. C 24, 1350021 (2013).

[9] Y. Ba, H. Liu, Q. Li, Q. Kang, and J. Sun, Phys. Rev. E 94, 023310 (2016).

[10] S. Leclaire, N. Pellerin, M. Reggio, and J.-Y. Trépanier, Int. J. Multiphase Flow 57, 159 (2013).

[11] T. Akai, B. Bijeljic, and M. J. Blunt, Adv. Water Resour. 116, 56 (2018).

[12] H. Liu, A. J. Valocchi, and Q. Kang, Phys. Rev. E 85, 046309 (2012).

[13] H. Liu, Y. Ju, N. Wang, G. Xi, and Y. Zhang, Phys. Rev. E 92, 033306 (2015).

[14] S. Leclaire, N. Pellerin, M. Reggio, and J.-Y. Trépanier, J. Phys. A 47, 105501 (2014).

[15] X. Xu, K. Burgin, M. A. Ellis, and I. Halliday, Phys. Rev. E 96, 053308 (2017).

[16] D. Kehrwald, Ph.D. thesis, Fraunhofer-Institut für Techno- und Wirtschaftsmathematik, 2002.

[17] A. K. Gunstensen and D. H. Rothman, Europhys. Lett. 18, 157 (1992).

[18] A. P. Hollis, I. Halliday, and R. Law, Phys. Rev. E 76, 026709 (2007).

[19] T. J. Spencer, I. Halliday, and C. M. Care, Phys. Rev. E 82, 066701 (2010).
[20] K. Burgin, J. Spendlove, X. Xu, and I. Halliday, Phys. Rev. E 100, 043310 (2019).

[21] M. Geier, A. Fakhari, and T. Lee, Phys. Rev. E 91, 063309 (2015).

[22] A. Fakhari, T. Mitchell, C. Leonardi, and D. Bolster, Phys. Rev. E 96, 053301 (2017).

[23] I. Halliday, A. P. Hollis, and C. M. Care, Phys. Rev. E 76, 026708 (2007).

[24] D. J. Holdych, D. R. Noble, J. G. Georgiadis, and R. O. Buckius, J. Comput. Phys. 193, 595 (2004).

[25] S. T. Zalesak, J. Comput. Phys. 31, 335 (1979).

[26] Y. H. Qian, D. D'Humières, and P. Lallemand, Europhys. Lett. 17, 479 (1992).

[27] T. Krüger, H. Kusumaatmaja, A. Kuzmin, O. Shardt, G. Silva, and E. M. Viggen, The Lattice Boltzmann Method: Principles and Practice (Springer, Berlin, 2017).

[28] P. L. Bhatnagar, E. P. Gross, and M. Krook, Phys. Rev. 94, 511 (1954).

[29] A. Kupershtokh, D. Medvedev, and D. Karpov, Comput. Math. Appl. 58, 965 (2009), .

[30] U. D’Ortona, D. Salin, M. Cieplak, R. B. Rybka, and J. R. Banavar, Phys. Rev. E 51, 3718 (1995).

[31] M. Sbragaglia, R. Benzi, L. Biferale, S. Succi, K. Sugiyama, and F. Toschi, Phys. Rev. E 75, 026702 (2007).

[32] D. David, F. Kuznik, K. Johannes, and L. Merlier, Comput. Fluids 116, 46 (2015).

[33] A. J. Wagner, Phys. Rev. E 74, 056703 (2006).

[34] M. Gross, N. Moradi, G. Zikos, and F. Varnik, Phys. Rev. E 83, 017701 (2011)

[35] D. Lycett-Brown and K. H. Luo, Phys. Rev. E 91, 023305 (2015).

[36] Z. Guo, C. Zheng, and B. Shi, Phys. Rev. E 65, 046308 (2002).

[37] Y. Sun and C. Beckermann, J. Comput. Phys. 220, 626 (2007).

[38] P.-H. Chiu and Y.-T. Lin, J. Comput. Phys. 230, 185 (2011).

[39] M. Ben Said, M. Selzer, B. Nestler, D. Braun, C. Greiner, and H. Garcke, Langmuir 30, 4033 (2014). 
[40] R. Folch, J. Casademunt, A. Hernández-Machado, and L. Ramírez-Piscina, Phys. Rev. E 60, 1724 (1999).

[41] E. Olsson and G. Kreiss, J. Comput. Phys. 210, 225 (2005).

[42] I. Ginzburg, D. d'Humières, and A. Kuzmin, J. Stat. Phys. 139, 1090 (2010).

[43] D. d'Humières, I. Ginzburg, M. Krafczyk, P. Lallemand, and L.-S. Luo, Philos. Trans. R. Soc. London A 360, 437 (2002).
[44] S. Leclaire, N. Pellerin, M. Reggio, and J.-Y. Trépanier, Int. J. Numer. Methods Fluids 77, 732 (2015).

[45] C. M. Pooley and K. Furtado, Phys. Rev. E 77, 046702 (2008).

[46] S. Leclaire, A. Parmigiani, O. Malaspinas, B. Chopard, and J. Latt, Phys. Rev. E 95, 033306 (2017).

[47] S. P. Thampi, S. Ansumali, R. Adhikari, and S. Succi, J. Comput. Phys. 234, 1 (2013). 